\title{
Note on Latent Roots and Vectors of Segments of the Hilbert Matrix
}

\author{
By H. H. Denman and R. C. W. Ettinger
}

As a by-product of work on condition numbers, maximum and minimum latent roots, and the corresponding vectors, have been calculated for the Hilbert segments of orders $4,6,8$, and 10 , to about 17 significant digits.

This supplements the data of Fairthorne and Miller [1] by including minimum latent roots and corresponding vectors, and increasing the accuracy. It also verifies their data (after rounding) except that one error was found (see next paragraph) in their published results.

A Hilbert segment of order $N$ is the matrix $\|1 /(m+n-1)\| ; m, n=1, \cdots, N$. Let $H$ represent a Hilbert segment, $T$ its inverse, $\lambda$ a latent root, and $V$ a corresponding vector. Then, e.g., $\lambda_{1}\left(H_{6}\right)$ is the largest latent root of the segment of order $6 ; V_{N}\left(T_{6}\right)$ is the vector corresponding to the smallest latent root of the inverse of $H_{6}$. The error in Fairthorne and Miller's article occurs in the third element of $V_{1}\left(H_{6}\right)$.

The power method [2] was used, with a double-precision floating-point routine on the IBM 650. Smallest latent roots were obtained as $\lambda_{N}\left(H_{N}\right)=1 / \lambda_{1}\left(T_{N}\right)$, and verified (to as many significant figures as the method allows) by direct calculation of $\lambda_{N}\left(H_{N}\right)$ by the power method, $\lambda_{N}(H)$ being obtained as $\lambda_{1}(H-p I)$, where $I$ is the identity matrix and $p$ is slightly greater than $\lambda_{1}(H)$. The $N$ th vectors are given as $V_{N}\left(H_{N}\right)=V_{1}\left(T_{N}\right)$, because the method gives greater accuracy here for $V_{1}$ than for $V_{N}$ and because $T$ has no input error.

Terminal digits are uncertain by not more than one, as indicated by convergence rates.

$$
\lambda_{1}\left(H_{4}\right)
$$

1. 50021428005924281

$$
V_{1}\left(H_{4}\right)
$$

1.

0. 57017208366323583

0. 40677898802752924

0. 31814096887379396 $\lambda_{1}\left(H_{6}\right)$

1. 6188998589243391 $V_{1}\left(H_{6}\right)$

1

0. 5886285434255432

0. 4283272844289561

0. 3396618918387095

0. 2825235879421492

0. 2423378111228495 $\lambda_{1}\left(H_{8}\right)$

1. 6959389969219494

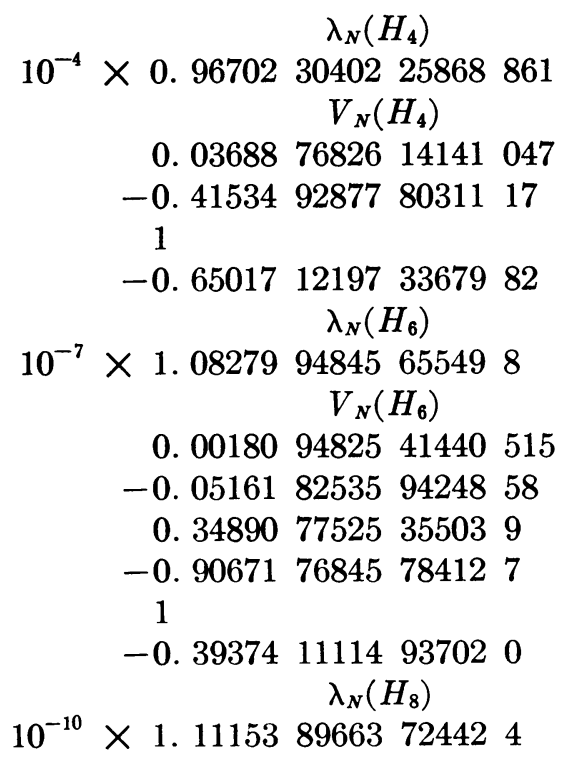

Received July 17, 1961. 


$$
V_{1}\left(H_{8}\right)
$$

1.

0. 6005042457579538

0. 4426715540119186

0. 3543704469996978

0. $296918578444507 \quad 1$

0. 2561809294869805

0. 2256293688082276

0. 2017901870379183

$$
\lambda_{1}\left(H_{10}\right)
$$

1. 7519196702651775

$$
V_{1}\left(H_{10}\right)
$$

1.

0. 6089919143696503

0. 4531382989594215

0. 3652860134021510

0. 3077530474455016

0. 2667251842930508

0. 2358013079824843

0. 2115639639515401

0. 1920051281861191

0. 1758600343931029

$$
V_{N}\left(H_{8}\right)
$$

-0.00006 861039214512812

0. 003687877051827661

-0.04826725452449843

0. 2617133996761041

-0. 7057473471796188 1.

-0. 7125091381801248

0. 2012418343837764

$$
\lambda_{N}\left(H_{10}\right)
$$

$10^{-13} \times 1.0931538198576599$

$$
V_{N}\left(H_{10}\right)
$$

0. $0000027147 \quad 13133604097$

$-0.0002360612629590383$

0. 005052897386716890

-0.04611604004998925

0. 2206615177289104

-0.6081767839543368

1 .

$-0.9681588795122191$

0. $509073585167138 \quad 3$

-0. 1121049402147474

Wayne State University

Detroit 2, Michigan

1. R. A. Fairthorne \& J. C. P. Miller, "Hilbert's double series theorem and principal latent roots of the resulting matrix," $M T A C$, v. 3, 1949, p. 399 ;

2. Marvin Marcus, "Basic theorems in matrix theory," Nat. Bur. Standards, Appl. Math. Ser. No. 57, U. S. Government Printing Office, Washington, D. C., 1960. 